Onkologe $2019 \cdot 25: 728$

https://doi.org/10.1007/s00761-019-0615-0

Online publiziert: 11. Juni 2019

(c) Springer Medizin Verlag GmbH, ein Teil von Springer Nature 2019
Hakan Alakus ${ }^{1}$ Michael Korenkov' · Fabian Kütting ${ }^{2} \cdot$ Christiane Bruns $^{1}$

${ }^{1}$ Klinik und Poliklinik für Allgemein-, Viszeral- und Tumorchirurgie, Universitätsklinikum Köln (AöR), Köln, Deutschland

${ }^{2}$ Klinik für Gastroenterologie und Hepatologie, Universitätsklinikum Köln, Köln, Deutschland

\section{Erratum zu: FOLFIRINOX beim duktalen Adenokarzinom des Pankreas}

\section{Erratum zu:}

Der Onkologe 2019

https://doi.org/10.1007/s00761-019-

0589-y

In der Originalversion des Beitrags wurde der Nachname des Zweitautors falsch geschrieben. Der Autor heißt korrekt Michael Korenkov. Die Originalversion des Beitrages wurde korrigiert.

\section{Korrespondenzadresse}

\section{PD Dr. med. Hakan Alakus}

Klinik und Poliklinik für Allgemein-, Viszeralund Tumorchirurgie, Universitätsklinikum Köln (AöR)

Kerpener Str. 62, 50937 Köln, Deutschland

hakan.alakus@uk-koeln.de 